(cause of Bacterial Vaginosis) was found to top the list. Also, unprotected sex during first sexual intercourse and multiple sexual partners are found to be the major risk factors for the wide spread of Bacterial vaginosis among the study's young women.

Method Bacteriological and differential laboratory tests (microscopic examination, normal physiological saline wet-preparation, Potassium Hydroxide $(\mathrm{OH})$ solution, whiff test, the litmus paper/PH level test, and gram staining) were carried out on High vaginal swab (HVS) specimens collected aseptically within 6 months from nonpregnant, non-menstruating young women who are not on antibiotics for a week before specimens were collected, and whose consent is sought before the study. The Study population is made up of both STD center attending and non-attending young women (mean age $=25.50)$. In addition, a standard questionnaire $(r=0.77)$ was administered to answer the major research/study questions on the major risk factors for Bacterial vaginosis.

Result From the 200 HVS collected, $68 \%$ of primary isolates were isolated: G vaginalis (51.72\%), C albicans (41.92\%), and $T$ vaginalis $(06.36 \%)$ respectively $(p<0.0001)$. According to the result from the study questionnaire, among 200 respondents, $37.71 \%$ had their first sexual intercourse without any protection, $39.16 \%$ were protected during their first sexual intercourse, and $23.13 \%$ did not recollect. Also, $31.65 \%$ had engaged in sexual intercourse with more than one sexual partner before with no protection in few occasions, $37.21 \%$ had had multiple sexual partners before with protection, $31.14 \%$ declined to provide answer to the multiple sexual partner questions. Conclusions Bacterial vaginosis was found to be the most prevalent among young women in urban cities in Nigeria, and the major risk of contracting the infectious disease is unprotected sexual intercourse with multiple partners, and during first sexual intercourse. However, there is need for effective and efficient sex education and awareness to encourage the partners to be faithful to one another and for the promotion of safe sexual intercourse by using preventive means such as condoms.

\section{P3-S7.04 CANDIDA SUSCEPTIBILITY TO ANTIFUNGALS IN GOMEL REGION (BELARUS)}

doi:10.1136/sextrans-2011-050108.487

\section{A Khaikova. Gomel State Medical University, Gomel, Belarus}

Candidiasis is a fungal infection that often affects urogenital tract. About $75 \%$ of women had at least one episode of vulvovaginal candidiasis. Recent times chronic cause of candidiasis is prevalent in the clinical picture of the disease in Belarus. Along with Candida albicans other yasts (Candida crusei, Candida tropicans, Candida glabrata, Candida parapsilosis, etc) play a role in pathogenesis of the disease. Wide use of fluconazole in Belarus as well as prophylactic treatment during prolonged antibiotic use caused the grow of the resistance to the drug. Aim of our study was to reveal the incidence of urogenital candidiasis in patients who where checked up for STI in Gomel dermatovenereal hospital (Belarus), to study the resistance of Candida spp. to nystatin, clotrimazole, and fluconazole in vitro. Materials and Methods We observed 4397 patients using clinical examination, microscopy, and cultural examination. Susceptibility to antifungal was studied by cultured method in vitro in 187 samples from the patients.

Results Urogenital candidiasis clinically and was diagnosed in 473 patients $(10.8 \%)$ and confirmed by laboratory tests. Susceptibility to nystatin was founded in 183 cases (97.8\%), to clotrimazole-in 125 cases $(66.8 \%)$. Susceptibility to fluconazole was much lower and was registered only in 37 cases (19.8\%).

Conclusions Candidiasis is a current problem for gynaecologists and venreologists in Belarus. Its rate reaches $10 \%$ among all patients coming for follow-up for STI. Effective treatment for candidiasis should be developed. Wide use of fluconazole should be reevaluated because of its low susceptibility.

\section{P3-S7.05 PHENOTYPIC CHARACTERISATION OF CLINICAL TRICHOMONAS VAGINALIS ISOLATES AMONG AAWSW IN SEXUAL PARTNERSHIPS}

doi:10.1136/sextrans-2011-050108.488

${ }^{1} \mathrm{C}$ Muzny, ${ }^{1} \mathrm{C}$ Rivers, ${ }^{2} \mathrm{~L}$ Mena, ${ }^{1} \mathrm{~J}$ Schwebke. ${ }^{1}$ University of Alabama, Birmingham, USA; ${ }^{2}$ University of Mississippi, Medical Center, Jacksonville, USA

Background Trichomonas vaginalis (TV) is the most common nonviral sexually transmitted infection in the world. TV prevalence in US women is consistently highest among African Americans (AA). However, little data exists on the risk of TV infection among women who have sex with women (WSW) and the mechanism of transmission is poorly understood. The goal of this study was to evaluate the concordance of TV isolates among AAWSW involved in sexual partnerships using the random amplified polymorphonuclear DNA (RAPD) technique.

Methods AAWSW involved in sexual partnerships and participating in a cross-sectional study of STI prevalence rates at the Mississippi State Department of Health STD clinic in Jackson, MS, were selected for this study if both women in the partnership were infected with TV. All women completed a confidential survey asking detailed sexual history questions about female and male partners during the past 12 months. The RAPD technique utilising six unique PCR primers was performed on TV clinical isolates from each sexual partnership. RAPD amplicons were collated and categorised to determine genetic similarity between isolates from paired couples.

Results TV isolates from three paired couples were included in this study. RAPD profiling demonstrated that only one out of the three pairs of TV isolates was concordant see Abstract P3-S7.05 figure 1.

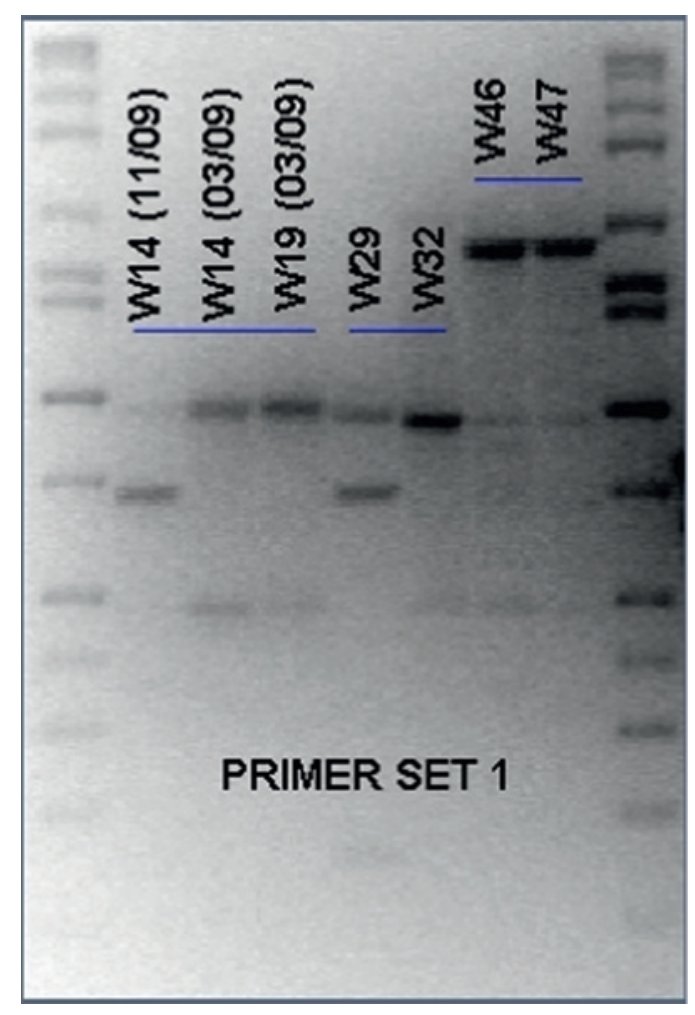

Abstract P3-S7.05 Figure 1 Primer Set 1. 